\title{
Ferroelectrics for Biomedical Applications
}

\author{
Fei Sun ${ }^{1}$, and Deyang Chen ${ }^{2 *}$ \\ South China Normal University, China
}

Submission: August 3, 2017; Published: August 31, 2017

*Corresponding author: Deyang Chen, South China Normal University, China, Tel: 86-13826413159, Email: dychen1987@gmail.com

Abstract

Ferroelectric materials, one of the current research focus with a number of fascinating properties such as ferroelectricity, piezoelectricity, pyroelectricity and dielectricity, has extensive application value in biomedical systems. Here we review the biomedical applications of ferroelectric materials, including ferroelectric ceramics, ferroelectric thin films, and ferroelectric polymers. Some unresolved problems are summarized and the future directions of applications in biomedicine are prospected as well.

Keywords: Ferroelectric ceramics; ferroelectric thin films; ferroelectric polymers; biomedicine; ultrasonic transducers

\section{Introduction}

Currently, researchers are mainly focusing the phase transitions, domain structures, domain wall functionalities, magneto electric coupling, and the potential applications in high-density ferroelectric non-volatile memories of ferroelectric materials [1-5]. However, there are relatively less research interests in the biomedical applications of ferroelectric materials which is very important to human life, such as the lead zirconate titanate (PZT) ceramics used in ultrasonic transducers for medical ultrasound systems and ferroelectric thin film capacitors implanted to the body as energy storage supply power for medical micro-devices. In this mini-review, we will introduce the biomedical applications of ferroelectric ceramics, ferroelectric thin films and ferroelectric polymers.

\section{Ferroelectric ceramics}

Ferroelectric ceramics, due to its piezoelectricity, pyroelectricity and dielectricity, are developing rapidly recently. The research directions of ferroelectric ceramics are wide and we focus on the introduction of its applications in biomedicine here.

\section{PZT ceramics}

Since lead zirconate titanate (PZT) of perovskite structure was discovered, it has been the most widely used ferroelectric ceramics because of its strong and stable piezoelectricity. In recent years, the PZT ceramics have a greater potential in application of ultrasonic transducers for medical ultrasound systems which are one of the basic imaging modalities covering cardiology, radiology, obstetrics, gynecology and general abdominal imaging. Piezoelectric materials are the core part of the ultrasonic transducer. They can generate a certain frequency of ultrasonic signals to detect some characteristics of a target media. Then they receive the signals containing information of the media and convert ultrasound signals into electrical signals. Piezoelectric phenomenon is that when a voltage is applied on the piezoelectric materials, the materials will elongate or contract. Reciprocally, they will generate voltages when an external mechanical force presses or elongates on them. Based on the high electro-mechanical conversion efficiency, PZT ceramics are extensively used in biomedical systems.

Recently, many other advanced ceramics have been developed rapidly. Chen et al. [6] reported the use of extrusion technique to fabricate transparent lead lanthanum zirconate titanate (PLZT) ceramic fibers. The final transducer was housed in an SMA connector for poling and pulse-echo measurement. The ceramic fibers can been used to create the small aperture ultrasonic transducer. And it has a great potential in photoacoustic imaging by using the transparent fiber as the path of light propagation and ultrasonic transducer materials.

Researchers also [7] investigated the design, fabrication, and experimental evaluation of a prototype PZT matrix transducer with an integrated receive Application Specific Integrated Circuit (ASIC) on what consists of an array of $9 \times 12$ piezoelectric 
elements mounted via an integration scheme, as a proof of concept for a miniature three-dimensional transesophageal echocardiography probe. The designs are employed to meet the strict space and power constraints, and demonstrated the effectiveness of the proposed techniques.

As the piezoelectric coefficient and electromechanical coupling coefficient of relaxor ferroelectric single crystal (such as lead magnesium niobate-leadtitanate (PMN-PT) and lead zinc niobate-leadtitanate (PZN-PT)) are more excellent than PZT, DeAngelis et al. [8] described a new effective method to explore lead indium niobate-lead magnesium niobate-leadtitanate (PINPMN-PT) transducer designs. The major advantage for PIN-PMNPT is that it can minimize the volume of piezoelectric material for the same impedance, and also allow larger transducers for a given operating frequency due to lower wave speed $\mathrm{c}$ and the handling durability or crack resistance was found to be similar to PZT8 with transducer build and rebuild.

However, there are still some problems to be solved, such as lead will cause harm to human and environment. It is crucial to look for the right elements to replace lead. Therefore, it is necessary to study the lead-free materials for biomedical applications such as $\mathrm{BiFeO}_{3}, \mathrm{BaTiO}_{3}, \mathrm{LiNbO}_{3}$ and relax or ferroelectric single crystals, etc [9-11].

\section{Bismuth-layered ferroelectric ceramics}

Bismuth-layered ferroelectric ceramics (BLF) have aroused much attention as a lead-free ferroelectric ceramics. Usually, BLF can be expressed as $\left(\mathrm{Bi}_{2} \mathrm{O}_{2}\right)_{2}+\left(\mathrm{An}-1 \mathrm{BnO}_{3} \mathrm{n}+1\right)_{2}^{-}$, wherein $\mathrm{A}$ represents $+1,+2$ or +3 valence ions and $B$ represents $+3,+4$ or +5 valence ions. $\mathrm{A}\left(\mathrm{An}-1 \mathrm{BnO}_{3} \mathrm{n}+1\right)_{2}$ - layer of a pseudo-perovskite structure is inserted between the $\left(\mathrm{Bi}_{2} \mathrm{O}_{2}\right)_{2}+$ layers. Strontium bismuth tantalate (SBT), a bismuth-layered ferroelectric material, has been found that it has a larger remaining polarization, good thermal stability performance and high Curie temperatures and it is widely used for sensors and memories [12]. However, it contains the volatile element, bismuth, which is easy to cause the formation of oxygen vacancies to deteriorate the anti-fatigue performance and ferroelectricity.

Much effort has been made to improve the related properties for the realization of biomedical applications. Zhou et al. [13] described an approach by adding $\mathrm{Al}_{2} \mathrm{O}_{3}$ to $\mathrm{Na} 0.5 \mathrm{Bi}_{2} \cdot 5 \mathrm{Nb}_{2} \mathrm{O}$ based bismuth layered piezoceramics to improve ferroelectric, piezoelectric and high temperature resistance. The bismuthlayered piezoceramic $\mathrm{Na} 0.5 \mathrm{Bi}_{2.5} \mathrm{Nb} 209-\mathrm{Al}_{2} \mathrm{O}_{3}$ shows large piezoelectric constant $\left(\mathrm{d}_{33}=15.2 \mathrm{pC} / \mathrm{N}\right)$, a relatively large remnant polarization $\left(\sim 11.6 \mu \mathrm{C} / \mathrm{cm}_{2}\right)$ and good temperature stability $\left(600^{\circ} \mathrm{C}\right)$, promising for future sensor applications and high temperature applications.

Sam et al. [14] used the solid-state reaction method to prepare intergrowth bismuth-layered ferroelectric(BLF)-type BiT-CaBi $\mathrm{Ti}_{4} \mathrm{O}_{15}$ (CBTO), BLaT-CBTO, and BNdT-CBTO ceramics.
The remanent polarization and piezoelectric coefficient values of the intergrowth ceramics are slightly larger than the BLF ceramics. After doping with rare-earth ions La and $\mathrm{Nd}$, i.e., the dielectric constants and the piezoelectric coefficients were improved to 146 and $15.4 \mathrm{pC} / \mathrm{N}$, respectively. On this basis, novel ferroelectric capacitor will be exploited for non-volatile memory storage and biomedical tactile sensor applications.

\section{Ferroelectric thin films}

With the continuous development of system integration and device miniaturization, ferroelectric thin films are gaining increasing attention for biomedical systems. For instance, ferroelectric thin film capacitor as an energy storage supply power for medical micro-device implanting into the body. Its high energy storage density, small volume and long service life make a great significant. Compared with ferroelectric ceramics, the ferroelectric thin films is more sensitive to pressure and the ferroelectric thin film technologies are a breakthrough technology for a new class of thin film ultrasonic transducers [15]. Seiya Ozeki et al. [16] synthesized PZT polycrystalline film via a hydrothermal method which is used for transducers to replace the conventional piezoelectric ceramic transducers, and succeed to reduce the size of PZT polycrystalline film vibrator for Coiled Stator Ultra-Sound Motor (CS-USM) with similar size as the piezoelectric ceramic resonator for CS-USM.

The elements of array of transducers can be defined only by the top electrode pattern, since the low lateral coupling of the printed PZT leads to a low cross-talk between the elements. It is necessary to apply PZT thick film technology to manufacture multi-element transducers enabling a novel method for costeffective fabrication of imaging arrays for medical applications. Bierregaard et al. [17] fabricated the transducers using screen printing technique, where the gold bottom electrode has been deposited first followed by the deposition of the PZT thick film; then the top electrodes define the elements of the array. The measured properties indicate very good reproducibility and repeatability within as well as between the devices. In addition, Lee et al. [18] made a diaphragm-type piezoelectric resonator for an operating flexure mode fabricated with thinned bulk PZT of a $50-\mu \mathrm{m}$ thickness bonded onto a silicon plate that was then strongly bonded onto a PDMS substrate using an oxygen-plasma treatment. This Flexible piezoelectric micro machined ultrasonic transducer (pMUT) array can be applied to study utilizing ultrasound brain stimulation.

\section{Ferroelectric polymers}

Ferroelectric polymers can not only turn mechanical, heat, sound vibration energies into electricity to harvest human mechanical energy, but also be used for pressure and temperature sensors due to their piezoelectricity, pyroelectricity, ferroelectricity and electrostrictive effect. Polyvinylidene fluoride (PVDF), polyvinyl fluoride and several copolymers are ferroelectric polymers with strong piezoelectric and pyroelectric 


\section{Global Journal of Nanomedicine}

properties. However, only cold drawing or electric polarization can enable PVDF show strong piezoelectric and pyroelectric performances. Otherwise, PVDF will miss these physical properties. Unlikely, the copolymers behave strong piezoelectric and pyroelectric as steric factors result in the mechanism of ferroelectric.

Fortunately, PVDF is a flexible materials and has been used as the medical ultrasonic transducers because of smaller decay time and more depth resolution than piezoelectric ceramics sensors. Salvatore A. Pullano et al. [19] presented a ferroelectric polymer-based temperature sensor by using a uniaxially stretched $28 \mu \mathrm{m}$ thick sheet of PVDF. The sensor made into a chip for micro fluidic devices can monitor the localized temperature of a biological fluid quickly. In terms of energyharvesting devices, their performances are relevant to the types of nanostructures of ferroelectric polymers.

Wang et al. [20] used a PVDF nanowire-PDMS composite film as the turboelectric layer, a polarized PVDF film as both the piezoelectric and pyroelectric layers to form a hybridized nanogenerator, which can harvest mechanical and thermal energies by turboelectric-piezoelectric-pyroelectric effects. These works extend the energy sources of the ferroelectric polymers and is attractive for biomedical applications.

\section{Conclusion}

In this mini-review, we give a brief summary of ferroelectric ceramics, ferroelectric thin films and ferroelectric polymers for the biomedical applications, including PZT ceramics used in ultrasonic transducers and ferroelectric thin film capacitors used as energy storage supply power for medical micro-devices. Besides, we propose possible future directions of this field, such as the study of lead-free materials for biomedical applications. We hope this review will arouse the readers' interest in this field.

\section{References}

1. Zeches R J, Rossell M D, Zhang J X, Hatt A J, He Q et al. (2009) A StrainDriven Morphotropic Phase Boundary in $\mathrm{BiFeO}_{3}$. Science 326(5955): 977-980.

2. Chen D, Chen Z, He Q, Clarkson JD, Serrao CR, et al. (2017) Interface Engineering of Domain Structures in $\mathrm{BiFeO}_{3}$ Thin Films. Nano Lett 17(1): 486-493

3. Seidel J, Martin LW, He Q, Zhan Q, Chu YH, et al. (2009) Conduction at domain walls in oxide multiferroics. Nature Mater 8 (3): 229-234.

4. Heron J T, Bosse J L, HeQ Gao Y, Trassin M, et al. (2014) Deterministic switching of ferromagnetism at room temperature using an electric field. Nature 516(7531): 370-373.

5. Chen D, Gao X, Liu J-M (2016) Domain structures and magnetoelectric effects in multiferroic nanostructures. MRS Commun 6(4): 330-340.

6. Chen X, Chen R, Chen Z, Chen J, Kirk Shung K, et al. (2016) Transparent lead lanthanum zirconate titanate (PLZT) ceramic fibers for highfrequency ultrasonic transducer applications. Ceram Int 42(16):1855418559.

7. Chen C, Raghunathan SB, Yu Z, Shabanimotlagh M, Chen Z, et al. (2016) A Prototype PZT Matrix Transducer With Low-Power Integrated Receive ASIC for 3-D Transesophageal Echocardiography. IEEE T ULTRASON FERR 63(1):47-59.

8. DeAngelis D A, Schulze G W (2015) Performance of PIN-PMN-PT single crystal piezoelectric versus PZT8 piezoceramic materials in ultrasonic transducers. Phys Procedia 63: 21-27.

9. Ascoli A, Senger V, Tetzlaff R, Du N, Schmidt O G, et al. (2016) BiFeO memristir-based encryption of medical data. IEEEInternational Symposium on Circuits \& Systems (ISCAS)DOI: 10.1109/ ISCAS.2016.7538871

10. Flores-Cuautle JJA, Suaste-Gómez E (2009) Developed of a novel ceramic-controlled piezoelectric of single disk of $\mathrm{BaTiO}_{3}$ for biomedical applications. Health Care Exchanges, Pahce Pan American DOI: 10.1109/PAHCE.2009.5158347

11. Dahmardeh M, Sheybanifar S, Janmaleki M, Abdolahad M (2015) Acoustic wave based biosensor to study electroacoustic based detection of progressive (SW-48) colon cancer cells from primary (HT29) cells. Sensor Actuat A-Phys 233(4): 169-175.

12. Fujimoto Y, Nakamachi E, Morita Y (2017) Biocompatible Aurivilliuslike layered ferroelectric $\mathrm{BaIn}_{2} \mathrm{Ta}_{2} \mathrm{O}_{9}$. Ceram Int 43: 7278-7281.

13.Zhou Z, Liang R H, Shao X F, Huang R, Cheng G F, et al. (2017) Microstructure and electrical properties of 3-0 type composite of $\mathrm{Na}_{0.5} \mathrm{Bi}_{25} \mathrm{Nb}_{2} \mathrm{O}_{9}$-based bismuth-layered piezoceramics. Ceram Int Doi:10.1016/j.ceramint.2017.05.361.

14. Cho S Y, Choi G P, Bu S D (2017) Comparison between the Electrical Properties of Bismuth Layer-Structured and Intergrowth Bismuth Layer-Structured Ferroelectric Ceramics. J Korean Phys Soc 70(10): 934-938.

15. Esipov YV, Biryukov SV, Masychev SI, Mukhortov VM (2016) Development of piezoelectric dynamic deformation transducers based on hot polarization of lead zirconate-titanate ferroelectric films. Measurement Tech 59 (4): 423-427.

16. Ozeki S, Takeuchi S, Kurosawa MK (2015) Fundamental study on the miniture CS-USM by hydrothermally synthesized lead zirconate titanate polycrystalline film transducer for medical applications. Ultrasonics Symposium 30(1):1-4.

17. Bierregaard LM, Zawada T, Ringgaard E, Xu R, Bagge J P, et al. (2015) Cost-effective screen printed linear arrays for medical imaging fabricated using PZT thick films. Ultrasonics Symposium DOI: 10.1109/ ULTSYM.2015.0277.

18. Lee JH, Cho J, Ko K, Yoon ES, Park HH, et al. (2016) Tae Song Kim, Flexible piezoelectric micromachined ultrasonic transducer (pMUT) for application in brain stimulation. Microsyst Technol 23(7):23212328

19. Pullano SA, Mahbub I, Islam SK, Fiorillo AS (2017) PVDF Sensor Stimulated by Infrared Radiation for Temperature Monitoring in Microfluidic Devices. Sensors 17(4):1-13.

20. Wang SH, Wang ZL, Yang Y (2016) A one-structure-based hybridized nanogenerator for scavenging mechanical and thermal energies by triboelectric-piezoelectric-pyroelectric effects. Adv Mater 28(15): 2881-2887. 
This work is licensed under Creative Commons Attribution 4.0 License

DOI: 10.19080/GJN.2017.02.555599
Your next submission with JuniperPublishers will reach you the below assets

- Quality Editorial service

- Swift Peer Review

- Reprints availability

- E-prints Service

- Manuscript Podcast for convenient understanding

- Global attainment for your research

- Manuscript accessibility in different formats ( Pdf, E-pub, Full Text, Audio)

- Unceasing customer service

Track the below URL for one-step submission https://juniperpublishers.com/submit-manuscript.php 